\title{
Las facultades de comunicación como 'laboratorios de prueba y error’ en la enseñanza-experimentación del nuevo periodismo
}

\section{Communication Faculties as 'Trial and Error Laboratories' in the Teaching- Experimentation of the New Journalism}

DOI: $10.46932 / \mathrm{sfjdv2n3-062}$

Received in: May 1st, 2021

Accepted in: Jun 30th, 2021

\author{
Prof. Dr. Jesús Miguel Flores-Vivar \\ Universidad Complutense de Madrid \\ Facultad de Ciencias de la Información (Avda. Complutense s/n. 28040) \\ Departamento Periodismo y Nuevos Medios \\ Internet Media Lab \\ E-mail: jmflores@ucm.es \\ Profa. Dra. Ana María Zaharía \\ Universidad Rey Juan Carlos/Campus de Fuenlabrada \\ Departamento de Ciencias de la Comunicación y Sociología \\ E-mail: anamaria.zaharia@urjc.es
}

\section{RESUMEN}

El presente trabajo analiza los aspectos formativos que tienen las facultades de comunicación, las tendencias y las iniciativas que algunos centros están desarrollando como parte experimental del ecosistema del periodismo en Internet, cada vez más consolidado aunque con incertidumbres en sus modelos de negocio, narrativos e informativos. Fomenta el desarrollo de la investigación aplicada, a través de medialabs, de nuevos modelos periodísticos -sin menoscabo de los valores éticos y de la calidad en la redacción-, en las ilustraciones, correcciones y producción de materiales en plataformas digitales y multimedia. Propone una reflexión del periodismo, no sólo como profesión, sino, fundamentalmente, como disciplina científica, avalada y justificada su enseñanza en las Facultades de Comunicación.

En este contexto, las facultades con estudios de periodismo asumen un papel fundamental y de vital importancia en la formación de periodistas de nuevo perfil, con un tipo de conocimientos que obliga a una revisión permanente de los planes de estudio. La metodología para realizar este estudio se basa en la revisión bibliográfica, informes y estudios sobre la formación periodística en donde impera lo multimedia, lo digital y lo online. Se complementa con un estudio Delphi, realizado a profesores-investigadores y profesionales de periodismo. Con los resultados obtenidos se pretende ofrecer algunas reflexiones sobre la formación periodística más experimental para estudiantes que deberán atender las necesidades informativas de una nueva "Sociedad red" (Castells, 2006), en donde los ciudadanos atienden a una definición distinta del acceso a la información y a su derecho a estar informados con contenidos de calidad, lo que consolida nuevas vivencias como algo especialmente nuevo, conocido ya como los "New, new media" (Levinson, 2012).

Palabras clave: periodismo, laboratorios-medios, experimentación, tecnologías, docencia

\section{ABSTRACT}

his paper analyzes the educational aspects of communication faculties, trends and initiatives that some centers are developing as an experimental part of the ecosystem of journalism on the Internet, increasingly consolidated although with uncertainties in their business, narrative and informative models . It 
encourages the development of applied research, through medialabs, of new journalistic models - without prejudice to ethical values and quality in writing - in illustrations, corrections and production of materials on digital and multimedia platforms. It proposes a reflection of journalism, not only as a profession, but, fundamentally, as a scientific discipline, endorsed and justified by its teaching in the Faculties of Communication.

In this context, the faculties with journalism studies assume a fundamental and vital role in the training of new profile journalists, with a type of knowledge that requires a permanent review of the study plans. The methodology to carry out this study is based on the bibliographic review, reports and studies on journalistic training where multimedia, digital and online prevail. It is complemented by a Delphi study, carried betwin professors-researchers and journalism professionals. With the results obtained, it is intended to offer some reflections on the more experimental journalistic training for students who will have to attend to the informational needs of a new "Network Society" (Castells, 2006), where citizens attend to a different definition of access to information and their right to be informed with quality content, which consolidates new experiences as something especially new, known as the "New, new media" (Levinson, 2012).

KeyWords: journalism, medialabs, experimentation, technologies, teaching.

\section{INTRODUCCIÓN. CONTEXTUALIZACIÓN ACTUAL DEL PERIODISMO.}

En esta Era de datos e información, la sociedad está exigiendo a los medios informativos una adaptación de forma y contenido en sus publicaciones. La situación actual de las organizaciones de noticias, y por ende, del periodismo profesional exige más rigor y profesionalidad. Junto a ello, se demanda, también, una formación idónea y de calidad que esté aparejada con los procesos evolutivos marcados por las tendencias de las Tecnologías de la Información y Comunicación (TIC) y, concretamente, el empuje de Internet y la proliferación de información y datos masivos (Big data), que fagocitan el llamado periodismo de datos u "otras opciones que incluyen la publicación de contenido relacionado con la supervisión de métricas y la búsqueda de documentos recomendados" (Ribeiro, 2020) entre otras innovaciones. En este contexto, hemos entrado en una época de cambios vertiginosos en todos los ámbitos de la información que van desde las fuentes a los públicos, desde los géneros tradicionales a las nuevas tecnologías. Asistimos a ver cómo emergen los nuevos medios, en donde el protagonista es también el usuario como parte de la nueva audiencia.

Por otra parte, entornos emergentes del nuevo periodismo como La narrativa transmedia, el periodismo móvil, el periodismo inmersivo y el periodismo emprendedor, por citar sólo algunos, configuran un panorama en donde es necesario proponer y recomponer nuevos esquemas formativos en las facultades de comunicación, aunque sin que esto afecte a la concepción fundamental del periodismo.

El periodismo, con sus más de dos siglos de existencia, ha sido impactado por las TIC y modifica sus estructuras. En primer lugar, ya no es el oficio como nos inculcaban en el ejercicio práctico y que cualquier persona con estudios en este campo, o sin ellos, podía ejercerlo. El periodismo ya no es el refugio 
-o debería dejar de serlo- de todos aquellos/as que habiendo estudiando un campo de conocimiento y dedicándose profesionalmente a ello, en sus horas libre, se dediquen, también, al "oficio" de periodista. Es hora ya de la constitución y creación de verdaderos colegios profesionales que velen por los intereses de los miles de licenciados y graduados en esta rama de la comunicación que dedicaron años de aprendizaje en las aulas universitarias. ¿Puede acaso un licenciado en periodismo que ejerce profesionalmente como tal, dedicarse en sus horas libres al oficio de abogado sólo con saber algo leyes o de medico sólo con leer un vademécum? La respuesta, obviamente, es no. Entonces ¿Por qué se hace con el periodismo? ¿Acaso el tiempo de estudios no es el mismo que emplean abogados, psicólogos, sociólogos o arquitectos?

Cuando un campo del conocimiento es reconocido como disciplina científica, es un indicador de avance y evolución cultural de una sociedad. Así, por ejemplo, la Medicina que empezó siendo una práctica empírica y que alcanzó su mayor conocimiento en las aulas universitarias hasta convertirse en lo que hoy constituye, es sólo una muestra de ese avance (Flores, 2014). Obviamente, los estudios de la Ciencia médica no son comparables con los estudios de Periodismo o Comunicación. Pero, lo que no podemos dejar de lado es que ya nadie duda de que el periodismo se ha convertido en uno de los pilares fundamentales de la democracia, tal y como afirma Abdul Waheed Khan (2007) ${ }^{1}$ en el prólogo del estudio titulado "Model Curricula for Journalism Education":

"El periodismo y los programas educativos que permiten a las personas utilizar y mejorar sus destrezas periodísticas constituyen instrumentos esenciales para sustentar principios democráticos clave que son trascendentales para el desarrollo de todos los países” (Unesco, 2007)

Bajo este prisma, se debe debatir el estado actual de los estudios de periodismo y, específicamente, la idoneidad de los planes de formación que se dan en las Facultades de Comunicación o Escuelas de Periodismo. Para empezar, debemos partir del hecho de que el periodismo ha dejado de ser un oficio para convertirse en una profesión reglada (como la del abogado, sociólogo, filósofo o psicólogo) aunque muchos persisten en definirlo aun como oficio, ignorando que la $\mathrm{UNESCO}^{2}$ lo describe como disciplina profesional y del conocimiento. Por ello, es necesario adaptar la "receta comunicativa" de la formación periodística al cambio de paradigma como consecuencia de la evolución constante de Internet y otras tecnologías digitales o nos resignaremos a languidecer. Félix Ortega $(2013)^{3}$, profesor de la Universidad de Salamanca, afirma que:

\footnotetext{
${ }^{1}$ Abdul Waheed Khan es Subdirector General de Comunicación e Información, UNESCO.

${ }^{2}$ Organización de las Naciones Unidad para la Educación, la Ciencia y la Cultura.

${ }^{3}$ Félix Ortega es profesor de Comunicación audiovisual en la Universidad de Salamanca.
} 
"los tiempos han cambiado y la profesión de comunicador y periodista debe adaptarse al cambio de los hábitos de consumo y de rentabilidad de la nueva industria que se derivan de las tecnologías de la información y la comunicación. Renovarse y adaptarse, o languidecer y morir”.

Dado ese cuadro de mando, no debería sorprender que las inscripciones en programas de periodismo parezcan estar estancadas o en declive. Lee Becker, autor principal del estudio sobre la educación en periodismo realizado anualmente por el Centro James M. Cox Jr. para la Capacitación e Investigación en Comunicación de Masas Internacionales de la Universidad de Georgia, informó en agosto de 2014 que sus datos mostraban que las inscripciones en las escuelas de periodismo habían disminuido en 2013 precipitadamente en algunos de los programas de posgrado más prestigiosos del país, incluso cuando están mejorando en la mayoría de las otras disciplinas.

En España, un artículo publicado en el periódico ElDiario.es, informa que la Federación de Asociaciones de Periodistas de España (FAPE), la primera organización profesional de periodistas a nivel nacional y que cuenta con más de 19.000 asociados, decidió que no admitiría a quienes no estén en posesión del título de periodismo, independientemente de si están ejerciendo como tales. La decisión, que encierra un debate antiguo dentro de la profesión, ha levantado críticas en algunos sectores y ha provocado que la Asociación Española de Comunicación Científica (AECC) se desvincule de la federación.

El objetivo de esta medida, según declara a eldiario.es el presidente de la Federación, Nemesio Rodríguez, es "darle valor al título de periodismo y tratar el problema del intrusismo". Además, Rodríguez asegura que "en los últimos años, en las asociaciones apenas han entrado periodistas que no tienen la titulación".

Por tanto, los desafíos del periodismo que tenemos por delante son enormes. El estado del periodismo y de su formación en las escuelas de periodismo y facultades de comunicación, atraviesa una época de incertidumbre y expectación. Los informes sobre la profesión periodística en su etapa formativa en las universidades dibujan un panorama que preocupa de manera global a educadores, profesionales y a las propias organizaciones de noticias.

El presente artículo aborda conclusiones y un conjunto de propuestas para el futuro de la educación periodística que pueden sorprender a lectores y académicos, aunque esperamos, tambien, que provoquen discusiones, comentarios, críticas y por supuesto, incluso, un rechazo bien meditado y con argumentos de peso; y forma parte de la investigación "Ecología de medios y tecnologías emergentes: Cibercultura, Interdisciplinariedad e Investigación Aplicada. Estudio e Innovación de Modelos Informativos Multimedia y Digitales” (Ref. PR75/18-21619. Convocatoria 2018 Santander-UCM).

\section{HIPÓTESIS Y OBJETIVOS}


La universidad juega un papel fundamental y de vital importancia en la formación de periodistas de nuevo perfil, con un tipo de conocimientos que obliga a una revisión permanente de los planes de estudio. Pero, ¿Cuáles son los contenidos de esos planes de estudio para la formación de nuevos perfiles profesionales? Una primera hipótesis que intenta responder a esta variable se desprende de las acertadas iniciativas que vienen haciendo instituciones de prestigio con estudios de periodismo y comunicación como la Universidad de Columbia de Nueva York, la City University of New York (CUNY), la News University del Poynter Institute de Florida (EE.UU.), la City University de Londres o la Universidad de Birmingham (UK), en cuyas escuelas de periodismo se han adoptado cambios estructurales en los planes de formación, incorporando asignaturas hasta hace poco impensables en la enseñanza periodística.

Pese a los cambios de estas instituciones de referencia, surgen algunas incógnitas tales como ¿Es suficiente el cambio estructural en los planes de formación de los estudiantes de periodismo?, ¿Qué tipo de conocimientos se han de considerar en la nueva estructura de los estudios periodísticos?, ¿Cómo adaptamos las características de la cultura del "Big data", la narrativa transmedia o el emprendimiento a la nueva realidad mediática? ¿Tiene el docente los conocimientos prácticos necesarios para impartir los nuevos contenidos formativos? ¿Quién forma al formador? ¿Son los docentes parte de la nueva era formativa con herramientas y recursos multimedia?

Una segunda hipótesis es que la facultades de comunicación deben cumplir y asumir nuevos roles en la formación de periodistas que demanda, a escala global, una sociedad hiperconectada. Por ello, el objetivo que persigue este análisis contempla no solo estudiar y describir el funcionamiento del esquema formativo y los roles que han de asumir las facultades de comunicación españolas, sino, también, proponer contenidos que implique nuevos conocimiento que los estudiantes de periodismo han de poseer para crecer profesionalmente en la industria de las organizaciones de noticias (Flores, 2011: 28), lo que se traduce en convertir las escuelas y facultades en laboratorios de prueba y ensayo (o error) con el objetivo de dar/impartir una formación acorde con la adquisición de competencias necesarias para el ejercicio de la profesión periodística..

\section{METODOLOGÍA}

La metodología para realizar este estudio se fundamenta en la revisión bibliográfica existente sobre la formación periodística para una industria de la información en donde impera lo multimedia y digital. Se ha escogido esta técnica puesto que la investigación bibliográfica es la etapa de la investigación científica donde se explora qué se ha escrito en la comunidad científica sobre un determinado tema o problema: ¿Qué hay que consultar sobre los planes formativos en periodismo y cómo hacerlo? En un segundo plano, no menos importante, nos basamos en el análisis y estudios realizados por instituciones 
de prestigio como los informes del Tow Center for Digital Journalism, Nieman Lab, EducationShift, Pew Research Center, Knight Foundation o Searchlights and Sunglasses (blog de Eric Newton), los informes de Unesco (2007 y 2013); el Informe Anual de la Profesión Periodística de la FAPE, documentos que inducen a pensar que el cambio, aunque gradual, se viene realizando. Un tercer método empleado ha sido la entrevista. Para ello, se ha recurrido a la opinión, en primer lugar, de un profesional en activo de un importante periódico que demanda los nuevos perfiles profesionales y en segundo lugar, entrevista realizada a un profesor con más de diez años de experiencia en la enseñanza en diversas facultades de comunicación españolas e internacionales.

Con los resultados obtenidos ofrecemos algunas reflexiones sobre la formación periodística para una nueva sociedad en donde los ciudadanos atienden a una definición distinta de las anteriores y que consolida nuevas vivencias como algo especialmente nuevo, conocido ya como los "nuevos nuevos medios" (Levinson, 2012).

\section{HACIA UNA INNOVACIÓN EN LA FORMACIÓN PERIODÍSTICA}

Desde hace algunos años, cada vez más voces, tanto académicas como profesionales, se alzan para poner en entredicho el futuro del periodismo como profesión motivado por las consecuencias del impacto tecnológico. Junto a estas afirmaciones, en algunos casos, acertadas aunque exageradamente alarmistas, surgen los debates sobre la idoneidad de los planes de formación en las facultades de comunicación, circunstancia que obliga a una reestructuración o un giro radical de ciento ochenta grados en los contenidos curriculares. En contrapartida, emergen profesionales con nuevos conocimientos, adquiridos de forma autodidacta, quienes comienzan a diseñar su propio futuro, ilusionados con proyectos que permiten que la profesión siga existiendo más allá de los grandes medios.

\subsection{REPENSAR LA ENSEÑANZA DE PERIODISMO Y EL APRENDIZAJE EN UN MUNDO INTERACTIVO}

Las tecnologías digitales e interactivas han revolucionado la forma de utilización y consumo de contenidos de los medios en las últimas dos décadas. Como resultado de ello, las formas de ejercer el periodismo y la entrega de noticias han cambiado en una escala de ciento ochenta grados. Principales medios de comunicación tradicionales han luchado para mantenerse al día con estas transformaciones y sobrevivir en una sociedad que cambia rápidamente. Como consecuencia de ello, el mercado laboral del periodismo también está cambiando dramáticamente, lo que plantea graves cuestiones sobre el futuro de la enseñanza y formación del periodismo. Los temas que se platean a modo de debates en diversos foros profesionales y académicos, son, generalmente: 
1. El futuro para la enseñanza del periodismo en la era digital

2. ¿Cuál es el alcance y la naturaleza de los desarrollos curriculares en la enseñanza del periodismo a la luz de la transformación digital?

3. Modelos educativos que aporten los conocimientos y habilidades necesarias para afrontar los retos que plantea el nuevo paisaje mediático

4. Experiencias en la enseñanza del periodismo para aprender más acerca de la enseñanza y el aprendizaje en un panorama cambiante de los medios

5. Debates de viejas y nuevas demandas en el apartado de la educación y la enseñanza del periodismo en el contexto del cambio tecnológico

6. Nuevos enfoques, paradigmas y filosofías que han surgido en la educación en los últimos tiempos que pueden ayudarnos a entender / evaluar mejor el impacto de los cambios tecnológicos en la enseñanza del periodismo

7. Debates sobre tecnologías digitales que afectan a los medios tradicionales y a la formación y enseñanza del periodismo

8. Las tecnologías digitales en la construcción de las identidades de periodismo de potenciales periodistas, estudiantes de periodismo y profesores de periodismo

9. Áreas en la enseñanza del periodismo más afectados que otros por la digitalización tecnológica, la convergencia de los medios y la fragmentación de la audiencia

10. Papel de docentes de periodismo siguiendo las tendencias del sector para establecer y desarrollar los parámetros de la enseñanza y el aprendizaje de periodismo o explorar nuevas alternativas.

11. El papel de la investigación académica en periodismo para la enseñanza del periodismo a la luz de los cambios tecnológicos

Howard I Finberg $^{4}$ (2013), director de formación del Poynter Institute, en su artículo titulado “Repensar el periodismo. Una llamada a la innovación” publicado en el sitio web del Poynter, a raíz del Informe sobre el Estado de la Educación en Periodismo ${ }^{5}$, hace un diagnóstico de la situación de la formación periodística (2013):

"Lo que da miedo de una ruptura es que no sabe a dónde irá. Hace cuarenta años, no nos dábamos cuenta que la primera llamada de teléfono móvil llevaría todo lo móvil a la informática y los teléfonos inteligentes. Hace veinte años, no nos dimos cuenta de que Amazon transformaría compras al por menor. Hace diez años, no existía Facebook, ni Twitter. Usted simplemente no

\footnotetext{
${ }^{4}$ Director of Training Partnerships and Alliances The Poynter Institute for Media Studies

${ }^{5}$ El Estado de la Educación en Periodismo es el informe que anualmente elabora el University News del Poynter Institute. Florida, EE.UU.
} 
sabe a dónde nos conducirá la innovación disruptiva. Lo que sí sabemos, sin embargo, es que el futuro de la enseñanza del periodismo se encuentra en una situación crítica"

Finberg (2013), señala dos razones para argumentar su reflexión:

1. El tiempo se agota. La disrupción, impulsada por la economía y la tecnología, está realizándose mucho más rápido que la mayoría de los administradores del sistema universitario.

2. La educación en Periodismo sufrirá cambios fundamentales, sobre todo, en la forma como se enseña el periodismo y quien enseña. Los que no innovan en el aula, se habrán quedado atrás, al igual que los que optaron por no innovar en la sala de redacción.

Desde hace algunos años, se han desatado discusiones sobre el futuro de la formación en periodismo en las escuelas universitarias. Académicos y profesionales aún siguen debatiendo como se verá el futuro de la educación en periodismo, al igual que los profesionales de la información vienen discutiendo sobre cómo será el futuro del periodismo. El resultado de una encuesta del Poynter News University llevada a cabo en la primavera de 2012, destacaba que "muchos podemos sentirnos decepcionados, pero no sorprendidos de que las posiciones tanto para los educadores y los profesionales no han cambiado mucho en el último año”. Una nueva encuesta realizada por la misma institución(a finales de ese año), no muestra cambio alguno en las actitudes de ambos grupos. Con más de 1.800 respuestas, dividido entre profesionales y académicos, aún hay una gran distancia [más de 40 puntos] entre los dos grupos de encuestados.

Pese a no esperar ver un cambio masivo en las opiniones de los encuestados, sí se esperaba ver un poco movimiento, especialmente entre los académicos. De los resultados de dicha encuesta del Poynter, se destaca:

1. El 96 por ciento de los que se identifican como educadores (profesores) del periodismo creen que un grado en periodismo es muy importante. Y, extremadamente importante cuando se llega a la comprensión del valor del periodismo. Este resultado es casi idéntico al resultado de 2012. Los profesionales [editores, periodistas y otros relacionados] tienen una opinión menos favorable (57 por ciento) afirmando que un título sólo es valioso cuando se trata de entender el valor de periodismo. Este es el mismo porcentaje que en la encuesta de 2012.

2. Tampoco se perciben cambios en la brecha entre docentes y profesionales acerca de su visión sobre la importancia de un grado en periodismo cuando se trata de "habilidades en la recopilación 
de noticias, edición y presentación de las noticias". Casi todos los educadores (98 por ciento) dicen que un grado es extremadamente importante cuando se trata de las habilidades en la recolección de noticias. Pero sólo el 59 por ciento de profesionales comparte esta opinión, ya que casi uno de cada cinco dice que un título no es del todo importante o es sólo ligeramente importante en términos de recopilación de información de noticias. Existe una gran desconexión entre la profesión y la comunidad académica. Incluso, los docentes ven con preocupación de que la formación en periodismo no está a la par con lo que se hace en la profesión.

3. El treinta y nueve por ciento de los docentes ha manifestado que la educación en periodismo que busca mantenerse al día con los cambios de la industria hace nada o muy poco. Los líderes de las salas de redacción y miembros del personal son aún más críticos, con el 48 por ciento, diciendo que la academia no está al día con los cambios que el campo del periodismo demanda.

4. En cuanto a si un grado en periodismo es valioso cuando se trata de conseguir un trabajo, la brecha entre profesores y profesionales es más pequeña. Más de la mitad [53 por ciento] de los docentes piensan que un título en periodismo es muy importante a extremadamente importante para conseguir un puesto de trabajo. En contrapartida, sólo un cuarenta y uno por ciento de los profesionales comparten esa creencia. Pero aquellos que se identifican a sí mismos como "trabajo por cuenta propia" tienen una opinión aún más baja acerca de un grado de periodismo, y sólo un 38 por ciento dice que un título de periodismo es muy importante a extremadamente importante para conseguir trabajo.

Estos resultados significan que, a pesar de que las actitudes no han cambiado desde la encuesta del 2012, si existe una percepción de redoblar los esfuerzos para repensar la enseñanza del periodismo.

Una cuestión similar ya se había diagnosticado en el mencionado Informe Modelo de Enseñanza del Periodismo (Unesco, 2007), en donde se indica que uno de los puntos débiles de buena parte de la enseñanza del periodismo es consecuencia de la incapacidad de apreciar el grado en que la enseñanza de disciplinas universitarias constituye (junto a la cobertura periodística y la redacción) el fundamento del ejercicio del periodismo. En el otro extremo de esa ecuación se halla la necesidad de los estudiantes de periodismo de recibir formación sobre técnicas periodísticas y sobre el empleo del equipo impartida por periodistas competentes en ejercicio, no sólo como docentes invitados puntualmente sino como integrantes respetados (y debidamente retribuidos) del claustro de profesores. Es decir, una sinergia real entre el mundo de la academia y el mundo profesional. Un modo de enriquecer esta vertiente práctica, 
todos los programas de estudios de periodismo tendrían que incluir un periodo de prácticas (pasantías) en un medio de comunicación -u organización de noticias- y sería necesario que las escuelas (o facultades) de periodismo se dotaran de acuerdos de colaboración con medios de comunicación locales.

Al respecto, los convenios de colaboración entre organizaciones de noticias y universidades podrían contemplar, entre otras actividades, seminarios prácticos impartidos por profesores de periodismo en colaboración con periodistas de reconocido prestigio y, fundamentalmente, la estancia en medios de comunicación de miembros del claustro de profesores en comisión de servicio o intercambios y proyectos conjuntos. Las facultades de periodismo pueden organizar la celebración de seminarios en las sedes de los medios de comunicación con los que mantuvieran convenios con el objetivo de permitir a los estudiantes acceder a tecnología y servicios de información de los que carecen en la facultad. Esa colaboración puede servir para acortar la distancia entre los programas de estudios de periodismo y el sector de la industria de los medios de comunicación.

El Informe de la Unesco (2007) propone instar al sector de los medios de comunicación e información a que concedieran a los periodistas tiempo para poder estudiar o reciclarse en universidades o escuelas superiores y a que brindaran a los educadores -docentes- de periodismo la posibilidad de mejorar sus destrezas profesionales.

\subsection{ADAPTACIÓN A UNA CIBERCULTURA DEL CAMBIO PARA LA FORMACIÓN DE NUEVOS PERFILES}

En el estado actual del periodismo y los medios, urge una remodelación y revisión de los contenidos que se imparten en las facultades y escuelas de periodismo. La formación y aprendizaje del periodismo y comunicación en las aulas universitarias ha sido, durante décadas, un modelo que se ha basado en las clases presenciales. El aprendizaje y conocimiento sobre esta disciplina, se ha transferido, tradicionalmente, a través de clases magistrales impartidas por teóricos de la comunicación. En la actualidad, el modelo de aprendizaje y docencia del periodismo y comunicación sigue siendo el mismo, aunque a ese modelo tradicional se suman unos componentes que en solo diez años de existencia han irrumpido vertiginosamente, impactando en todas las esferas de la sociedad y del conocimiento. Estos componentes o recursos abanderadas por Internet y otras tecnologías emergentes afectan de lleno, no solo al contexto de la profesión periodística, sino a la forma y el fondo del proceso de enseñanza-aprendizaje en esta titulación.

Este nuevo modelo de enseñanza-aprendizaje, que se basa en el apoyo de los campus virtuales, no trata de sustituir las clases presénciales que se imparten en la licenciatura, grado o postgrado, sino, que su objetivo es, principalmente, convertirse en un complemento del aprendizaje que redunde en la formación 
del alumno, además de ser un apoyo complementario para la realización de tutorías más personalizadas y con mayor capacidad de respuesta. Así, el uso de internet y otras tecnologías emergentes, están siendo estudiadas y aplicadas a la docencia de forma progresiva. Por ejemplo, el uso de plataformas blogs o wikis para la generación de interactividad y participación en red y la telefonía móvil con sus nuevos códigos de comunicación.

En la sociedad industrial tradicional la educación estaba íntimamente ligada a las posibilidades de movilidad social. Cuanto más alto se llegase en niveles educativos más fácil sería llegar a altos puestos en la jerarquía social. Una de las grandes motivaciones para que una persona estudiara venía dada por la posible recompensa del ascenso social. En la actualidad, llegar a la cúpula del sistema educativo no implica acceder a la cúpula del sistema social. Es decir, tener un título universitario no es sinónimo de acceder rápidamente a un puesto de trabajo.

Por ello, los entornos de enseñanza/aprendizaje no pueden permanecer anquilosados en el pasado, en la forma tradicional de enseñanza. Esto significa la exigencia de nuevos roles tanto en profesores como estudiantes. La perspectiva tradicional en educación superior, por ejemplo, del profesor como única fuente de información y sabiduría y de los estudiantes como receptores pasivos debe dar paso a papeles bastante diferentes con características más activas. La información y el conocimiento que se puede conseguir en las redes telemáticas en la actualidad son ingentes. Cualquier estudiante universitario puede conseguir más información de la que su profesor tardará meses en disponer por los canales tradicionales. La misión del profesor en entornos ricos en información es la convertirse en facilitador, guía o consejero sobre fuentes apropiadas de información fomentando entre los estudiantes la creación de hábitos y destrezas en la búsqueda, selección y tratamiento de la información.

Las facultades o escuelas de periodismo a menudo han sido consideradas como las escuelas de comercio más que modernas escuelas profesionales. Como indicamos en otros apartados, para algunos medios de comunicación que han creados sus propias escuelas o centros de formación, estos son unidades de negocio. Como la importancia del periodismo crece y su tarea de explicar el mundo para el público se vuelve más complejo y exigente, las escuelas de periodismo deben moverse con firmeza en el ámbito de la escuela profesional. Esto incluye al personal docente, cuyos miembros deben ser profesionales con experiencia en medios o ser líderes activos en la profesión, demostrado a través de su trabajo periodístico, su enseñanza y su participación en el discurso público, lo que conferirá a los estudiantes no sólo la entradanivel de habilidades de trabajo, sino, también, un sentido de la historia de la profesión y su importancia para la sociedad y el Estado. Esta situación significa que las facultades de periodismo deben basarse en los recursos disponibles en sus universidades para dar a los estudiantes el conjunto más poderoso de posibles herramientas las que ayudarán a transmitir sus conocimientos al público. Las escuelas de 
periodismo están comprometidas con la idea de que las sociedades funcionan mejor cuando sus ciudadanos tienen acceso a la información que ha sido recopilada y presentada por profesionales muy bien formados, bien educados, honestos, dignos de confianza. En suma por personas inteligentes que han dedicado su vida a una noble profesión como es el periodismo.

\subsection{PROPUESTAS FORMATIVAS PARA NUEVOS PERFILES: EL PERIODISTA PROGRAMADOR}

Es hora de que los futuros periodistas sepan cómo trabajar con el código, como desarrollar una aplicación, como crear y diseñar. Asumiendo que se viene enseñando a los estudiantes de periodismo las técnicas del siglo pasado, es hora de innovar nuestros planes de estudio realizando un cambio de 180 grados. Pero, el aprendizaje de un software específico obliga a una formación continuada (Briggs, 2007). Nadie puede aprender narración digital en un semestre. Por ejemplo, el dominio de Dreamweaver, Flash o cualquier $\mathrm{CMS}^{6}$, no es que sea complicado su aprendizaje, pero tampoco hay que pensar que se aprende de "prisa y corriendo" subestimando la magnitud del conocimiento de este tipo de aplicaciones, por lo que incluir su aprendizaje en un curso de periodismo online o ciberperiodismo, como materia optativa puede hacer más daño que bien. Esto significa que debemos estar enseñando código en todos los cursos del grado de Periodismo (cada semestre, cada año, hasta la graduación).

Miranda Mulligan (2013), directora ejecutiva de Innovation Lab Knight News en Northwestern University y ex directora de diseño digital de The Boston Globe, afirma que los periodistas tienen que aprender cómo codificar si se quiere llegar a ser mejores narradores. Por ello, las escuelas deben enseñar a los estudiantes a tener una mente abierta acerca de sus habilidades- y eso significa aprender, al menos, lo básico de la programación. Con el conocimiento básico de la programación, los periodistas pueden aprender a crear aplicaciones (mashups) para contar historias, así como la forma de realizar presentaciones (visualizaciones) en forma de código con las noticias en "caliente” (actuales). Nunca antes ha sido más urgente para la industria de las noticias el entender el código lo suficientemente como para tener conversaciones significativas con los tecnólogos. En este contexto, uno de los retos que tienen los educadores-formadores-docentes consiste en eliminar el miedo de aprender, de reciclarse, un miedo notoriamente frecuente entre los periodistas. En gran medida hemos asistido a este tipo de debate y discusión una y mil veces. Pero, ahora, no sólo los periodistas visuales se quejan por el estancamiento de la narración y presentación en línea de donde surge una sola respuesta a esto: Los periodistas deben aprender más sobre el código. Entender y comprender el medio nos hace mejores narradores. Para una industria que se precia de ser inteligente y tolerante, ignorar a Internet puede conducir al fracaso (Flores, 2012).

\footnotetext{
${ }^{6}$ CMS es el acrónimo de Content Management System (Sistema de Gestión de Contenidos)
} 
La oferta de trabajos para diseñadores y periodistas que saben escribir código está creciendo exponencialmente. Por tanto, vamos a atacar el problema de la (pésima) formación desde todos los ángulos ya que, detectado el problema ahora toca encontrar la solución. En otras palabras: Tenemos que enseñar a los estudiantes a ser más tecnológicamente alfabetizados. Tenemos que enseñarles cómo aprender y cómo errar, bajo el modelo de prueba y error. Porque no es lo mismo ser nativo digital que alfabeto digital. Esto no significa que los redactores o editores de contenidos en blogs o páginas web, que han aprendido un poco de programación deben terminar por convertirse en un ingeniero de software o un diseñador web. Sencillamente, no. Tampoco el objetivo final es la fluidez en la programación de software, sino la creatividad en los contenidos de información. Sin embargo, hay un valor añadido en la comprensión de cómo los navegadores leen y procesan nuestras historias.

Por ello, a la cobertura periodística y redacción de una historia, se suman, el escribir algo de código (HTML, CSS, Javascript) y la programación de aplicaciones y servicios complejos, lo que convierte al periodista profesional en un perfil con toda una colección de habilidades. Un conocimiento fundamental de código permite:

- Mantener conversaciones cada vez más importantes sobre la presentación digital.

- La comprensión del medio te hace mejor en su oficio.

- Tener un pensamiento y comprensión de datos más profundo.

- Aprender más acerca de lo que sucede en la escritura digital y el software de programación enseña a pensar en términos de abstracciones, funciones, parámetros, componentes, marcos de trabajo, clases de objetos, plantillas y mucho más.

- En última instancia conduce a una mejor y más significativa narración de historias en línea.

El periodismo necesita graduados cualificados que puedan crear sofisticadas presentaciones visuales o aplicaciones mediante la utilización del código de la programación. Aunque, en un principio, pueda parecer aburrido, sin emoción y muchos estudiantes se sientan intimidados a la hora de enfrentarse al uso de código, esa aprehensión pasa cuando se dan cuenta que no es tan difícil. Es una situación que, seguramente, muchos docentes han tenido -y tienen- todos los semestres durante las prácticas de asignaturas de contenido práctico como ciberperiodismo o multimedia, teniendo que lidiar con el lenguaje HTML, CCS o el uso de aplicaciones CMS.

Con este enfoque formativo, podremos desbancar el sentimiento predominante que existe en las especialidades de periodismo y comunicación en cuyo entorno los periodistas se sienten aun intimidados por las tecnologías digitales (Bradshaw and Rohumaa, 2011). Y este sentimiento también es frecuente en 
las facultades de comunicación en donde se estudia periodismo. Por citar un ejemplo, en las clases de multimedia, el autor de este artículo se ha encontrado con estudiantes que mantienen cierta reticencia en el uso de la codificación en HTML. Por tanto, es el trabajo de los docentes-investigadores eliminar el miedo de aprender, un miedo notoriamente frecuente en los periodistas. El HTML no es magia. Escribir código no es magia, es sólo un trabajo duro. Aprender a programar no salvará al periodismo y probablemente no va a cambiar la forma en que escribimos nuestras historias. Sin embargo, es un hecho mucho más divertido ser un periodista en la web una vez y ver "cómo los ordenadores leen y entienden nuestro contenido".

Aprender a programar no sólo proporciona una habilidad práctica. También enseña a resolver problemas. Los estudiantes aprenden a ser más precisos, matizando procesos de pensamiento, por lo que la profundidad de su comprensión de la información y los datos sólo tiende a crecer. Además, los periodistas visuales, asumen que la enseñanza del código es la enseñanza del diseño de la información. Tanto los diseñadores de noticias, así como, los diseñadores de páginas web asumen las mismas responsabilidades: organizar racionalmente el contenido, lo que ilustra las ideas para profundizar en la comprensión de una historia y trabajar dentro de las limitaciones del medio.

Una de las tareas más importante que un docente siempre debe hacer es inspirar a los estudiantes a tener una mente abierta acerca de sus habilidades. Nadie sabe cómo se verá el paisaje de la narración en dos años y mucho menos en una década. Como instructores del aprendizaje, podemos hacer ver y hacer sentir a los estudiantes que convertirse en un periodista digital es una cuestión accesible y alcanzable. Los graduados deben salir preparados con un conjunto de habilidades que incluye la capacidad de aprender rápidamente y adaptarse a cualquier entorno, estar abierto a nuevas ideas y soluciones, y tomar la iniciativa. Este perfil de profesionales nunca se aburrirá y su conocimiento siempre puede ser -y será- el arma para combatir el desempleo. Nuestra pedagogía y la evangelización del nuevo periodismo basado en nuevos conocimientos, habilidades y destrezas, debería inspirar a futuros periodistas digitales a crecer continuamente y asumir que constantemente hay que aprender las herramientas que conllevan nuevas narrativas y técnicas, inculcando así, que los procesos de aprendizaje son de por vida. De ahí que, como afirmamos en otros apartados, el ejercicio del periodismo no debe concebirse como simple afición u oficio, sino como una carrera profesional, cuyos conocimientos se adquieren en las aulas universitarias. Y, desde una perspectiva científica, como una disciplina del conocimiento.

En este cambiante ecosistema mediático, para los periodistas y las universidades la mayor necesidad en su capacitación es como crear productos multimedia (Flores, 2009). En segundo término, cómo escribir para Internet y administración de comunidades online. En el mundo de hoy, de consumo y producción de noticias a gran escala, las escuelas de periodismo deberían, en mayor medida, estudiar los 
aspectos tecnológicos, posibilidades intelectuales, artísticas y literarias del periodismo y llevar una constante expansión y mejora de la capacidad de la prensa para informar al público, plena, profunda e interesante, acerca de asuntos públicos de importancia y complejidad.

Por todo esto, los planes de formación deben darse en función de las necesidades del mercado de la información y de lo que demanda la sociedad de cada país. La reestructuración de los planes formativos en las facultades de periodismo y comunicación debe hacerse a través de una encuesta marco a profesionales y académicos investigadores que responda sobre habilidades formativas o conocimientos más tecnológicos.

\subsection{PROPUESTAS PARA LA INCLUSIÓN DE CONTENIDOS INNOVADORES EN LOS ESTUDIOS DE PERIODISMO}

La detección de nuevos perfiles obliga a emprender nuevas líneas de formación, por ejemplo, en el ámbito de las redes sociales (Flores, 2009). Para ello, hace falta un análisis de los planes de estudios actuales de grado y posgrado con el fin de detectar las necesidades formativas no cubiertas. Esto reclama, por una parte, una concepción transversal: aspectos incluidos en el conjunto de asignaturas aunque no aparezca en su denominación referencia a las redes sociales y, por otra, una concepción focalizada exclusivamente en redes sociales y que puede aparecer en el título de la asignatura. Las líneas de enseñanza deben estar profundamente relacionadas con los perfiles profesionales emergentes y los que puedan apreciarse en el transcurso del tiempo. Los perfiles profesionales y la necesidad de enseñanza para cubrirlos forman tal unidad que reclaman mayor interrelación (Flores y Salinas, 2012). Reconociendo esta tendencia, programas de periodismo en las universidades de distintos países, incluido España, han estado tratando de renovar sus currículas de estudio con la intención de ofrecer cursos que enseñen, por ejemplo, a escribir para la Web o el uso de la narrativa transmedia y familiarizar a los estudiantes con software utilizado en el entorno multimedia, tales como HTML, Phyton (codificación esencial para algoritmos), Adobe Premiere Pro o Movie Maker (para editar video), Flash (para presentar video online y crear presentaciones interactivas) o CMS (Gestores de contenidos) para la construcción de sitios Web.

De ahí que la formación universitaria en la disciplina periodística pasa por incluir materias que antes estaban vedadas al conocimiento de los periodistas, tales como:

- Formación en periodismo y programación

- Formación multimedia y realidad inmersiva

- Formación en tratamiento y análisis estadísticos de datos

- Formación en Antropología y etnografía del periodismo en red 
En este contexto, diversos profesionales de medios, en concordancia con los académicos, proponen algunas iniciativas a tener en cuenta. Julio Miravalls $^{7}$ (2014) propone establecer cuatro áreas diferenciadas, en todas las cuales el estudiante de periodismo debería acreditar un conocimiento y capacitación al completar un currículo educativo en los años establecidos (sean cuatro o cinco). Estas cuatro áreas, son:

- Conocimientos generales teóricos:

Incluyendo las asignaturas clásicas de humanidades (geografía, arte, historia, literatura, religiones y filosofía), historia del periodismo, las propias de la nueva cultura (tecnologías, ciencia, cine, deporte...), más fundamentos de derecho, teoría política, ciencias económicas, administración de empresas, estadística, salud y medio ambiente, estructura política de España (o el país correspondiente, si hablamos de un planteamiento más amplio) y relaciones internacionales.

- Conocimientos teóricos especializados:

Desarrollos en mayor profundidad, con enfoques de conocimiento puro y de tratamiento periodístico de las materias más significativas, para poder ser elegidas como una o dos opciones específicas en el último periodo de los estudios (uno o dos cursos). El objetivo sería preparar posibles especializaciones en determinadas áreas de actividad o conocimiento mediante seminarios de alto nivel, talleres y trabajos muy elaborados de investigación y análisis.

- Estructura mental:

Aprendizaje y práctica intensiva de procesos intelectuales que no se basan estrictamente en el conocimiento teórico (aunque lo utilicen intensivamente) oratoria, lenguaje matemático, lógica, lenguaje estructural, semántica, diseño, programación informática (esto es una variante práctica que aglutina el lenguaje estructura y la lógica) e idiomas extranjeros (uno exigible con el máximo nivel y al menos otro, con un grado de comprensión y capacidades básicas de expresión). Se trata de crear un hábito de expresión, capacidad de comprensión (y evitar, de paso, el anumerismo que emborrona todo tipo de informaciones) y automatismos de reacción (improvisación y reflexión a la vez) ante cualquier circunstancia.

- Redacción:

\footnotetext{
${ }^{7}$ Julio Miravalls ha sido subdirector de ElMundo.es
} 
En el sentido más estricto y literal de la palabra. El estudiante de periodismo debería pasar a lo largo de la carrera muchísimas horas (digamos no menos de 300 por curso como media) de inmersión en una redacción real (o en la calle, trabajando para ella), desarrollando una doble actividad de aprendizaje teórico y ejercicio práctico profesional, del mismo modo que los estudiantes de medicina pasan horas diseccionando cadáveres (o viendo hacerlo) y acompañando a doctores en la visita a enfermos reales en los Hospitales Universitarios.

La propuesta de Miravalls, pasa por crear una redacción auténtica, que requeriría contar con el liderazgo de unos pocos profesionales con experiencia acreditada en el ejercicio del periodismo, para elaborar un producto informativo cotidiano (que puede estar disponible al gran público vía web, o de manera más restringida en las redes internas de la Universidad), con la exigencia de manejar con profesionalidad la actualidad y los hechos reales, trabajando en modo real en la captación y valoración de información, redacción, maquetación (diseño, escaletas...) y publicación.

Por su parte, Felix Ortega $(2014)^{8}$ afirma que "debemos adaptar la "receta comunicativa" al cambio de paradigma y a su rápida evolución o nos resignaremos a languidecer. Según Ortega, los tiempos han cambiado y la profesión de comunicador y periodista debe adaptarse al cambio de los hábitos de consumo y de rentabilidad de la nueva industria de las tecnologías de la información y la comunicación. Renovarse y adaptarse, o languidecer y morir.

\subsection{INVESTIGACIÓN APLICADA Y EXPERIMENTAL A TRAVÉS DE MEDIALABS O CENTROS DE INNOVACIÓN}

Desde hace algunos años, han empezado a proliferar los medialabs promovidos por las universidades y por los propios medios de comunicación, centrados en el futuro del periodismo y la esfera mediática por lo que corresponde observar qué tendencias se han descubierto en, por y para la profesión. Distintas universidades estadounidenses, europeas y españolas, vienen creando estos centros de investigación con objetivos similares (Flores, 2012: 7).

Iniciativas de esta naturaleza, vienen surgiendo cada vez más. Así por ejemplo, tenemos la propuesta hecha por la Fundación Nieman de la Universidad de Harvard, divulgando un sitioweb que lleva por nombre The Nieman Journalism Lab, y cuyo lema es bastante elocuente: "es un intento por ayudar al periodismo a descifrar su futuro en la era de la Internet”. En la misma línea de investigación sobre medios, pero con una visión global, se crea el MIT MediaLab. El MIT Medialab se define a sí

\footnotetext{
${ }^{8}$ Félix Ortega es Licenciado en Economía y Doctor en Comunicación y Cultura por la Universidad de Salamanca. En la actualidad, es profesor de la Facultad de Ciencias Sociales de la Universidad de Salamanca, España
} 
mismo como "El lugar donde el futuro se vive, no se imagina. En un mundo donde los avances técnicos están incorporándose, los investigadores del Media Lab diseñan nuevas tecnologías que ayuden a la gente a crear un mundo mejor”. Frank Moss (2011) actual director del MIT, afirma que:

\begin{abstract}
"Durante los últimos veinte años hemos sido pioneros en el diseño del estilo de vida digital del que disfrutamos hoy. Pero lo mejor aún está por llegar: una sociedad digital globalmente conectada que haga a la gente más feliz, más sana y más creativa. El laboratorio está liderando la investigación tecnológica que tiene como en ampliar y ensalzar las capacidades físicas, cognoscitivas y sociales de las personas. La tecnología abre la puerta a la creatividad, a la innovación y ayuda a resolver problemas -en particular los de la gente joven, nacida en la era digital-, algo que hasta ahora era imposible".
\end{abstract}

Las investigaciones que realizan instituciones y centros de investigación como el MIT Medialab abordan el entorno de los medios y del periodismo que, como disciplina del conocimiento y como reto profesional, los medialabs pueden aportar soluciones respondiendo a diferentes variables: ¿Cómo las nuevas tecnologías ayudan a mejorar las formas de comunicación? ¿Por qué la tecnología es beneficiosa para los nuevos profesionales del periodismo?, ¿Cuál es la formación práctica que necesitan los periodistas? etc.

Para Flores (2012: 9), los Medialabs se definen como entornos colaborativos de investigación y desarrollo de la convergencia de las nuevas tecnologías. Abarcan varios campos del conocimiento -como la informática, las comunicaciones, las nuevas tecnologías de la información, las ciencias sociales, la economía o el arte - y son laboratorios de ideas para instituciones y agencias públicas y privadas. Muchas de las innovaciones que han producido los medialabs han afectado, directa o indirectamente, a la profesión del periodismo. Los medialabs periodísticos suelen ser foros de discusión acerca de cómo la convergencia afectará a la profesión y qué nuevas tecnologías serán las que marquen las pautas de actuación en los próximos años.

\title{
5 CONCLUSIONES
}

Pese a los fallos del sistema universitario puntualmente detectados, la formación de los profesionales en periodismo que se da en la universidad española les permite competir en el mercado global de la comunicación al máximo nivel. Los profesionales se gradúan bien formados, aunque con ciertas carencias que exige el mercado actual de la información.

Por ello, debemos buscar que los nuevos entornos de aprendizaje del ciberperiodismo sean una forma de organizar el proceso de enseñanza presencial y a distancia que implica el empleo de tecnología, aunque no se reduzca solo a ello, por lo que hay que crear una situación educativa y metodológica centrada en el alumno, que fomente su auto aprendizaje, la construcción de su conocimiento y como parte de este proceso, el desarrollo de un pensamiento crítico y creativo. Estos aspectos son fundamentales en los 
procesos de enseñanza-aprendizaje del Espacio Europeo de Educación Superior y aplicable a todas las disciplinas del conocimiento, pero más en la disciplina del periodismo y comunicación.

Por otro lado, en concomitancia con el aspecto docente, las investigaciones demuestran que la generación de red, asentada en las aulas universitarias, no aprende de modo lineal y ponen en evidencia los distintos estilos de aprendizajes, todos válidos, que posee esta generación. Uno de los retos que los estudiantes de ciberperiodismo presentan a sus docentes es precisamente la atención personalizada que requiere. Exige el desarrollo de una serie de competencias profesionales, metodológicas, pedagógicas y didácticas para hacer posible que la universidad cumpla su cometido a la altura de las exigencias de una generación con extraordinarias potencialidades.

Las peculiaridades de la emergente generación que se expande cada vez más, parecen imbricados con una formula (generacional): "Con el ordenador e internet, todo. Sin ello, prácticamente, nada". Así de fácil o complejo resulta atender y satisfacer sus necesidades de aprendizaje y crecimiento, basado en el modelo de prueba y error que promueven los laboratorios de investigación. La generación red quiere aprender por vías no tradicionales y siempre empleando nuevas tecnologías. 


\section{BIBLIOGRAFÍA}

- $\quad$ Bradshaw, P. and Rohumaa, L. (2011) The Online Journalism Handbook: Skills to Survive and Thrive in the Digital Age. London, UK. Longman Practical Journalism Series

- $\quad$ Briggs, M. (2007) Journalism 2.0. Austin, EE.UU. Edt. Knight Center of Texas University

- Cebrián, M. (2005). La información multimedia. Soportes, lenguajes y aplicaciones empresariales. Madrid, Pearson Educación.

- $\quad$ El Diario.es (2019)"¿Debe un periodista tener la carrera de Periodismo?"

https://www.eldiario.es/sociedad/periodismo_1_1451891.html

- $\quad$ Flores, J.M.

_(2009). "Nuevos modelos de comunicación, perfiles y tendencias en las redes sociales". Comunicar, 33, 73-81. (DOI: 10.3916/c33-2009-02-007).

_ (2011) (ed.). Reinventar el Periodismo y los Medios. Madrid, Editorial Fragua

_ (2014) "El Periodismo como Disciplina del Conocimiento y el Valor Academicista para una Profesión de Calidad”. Prisma Social, no 12 pp. 506-543.

_(2012) "La importancia de los medialabs para la investigación aplicada con tecnologías emergentes. Estudio de caso: creación de mashups". En Periodismo en Red. Actas del V Congreso Internacional de Periodismo en Red. UCM. ISBN (CD-ROM) 978-84-615-6211-4 (V) 05-19.

- $\quad$ Flores, J.M. y Salinas, C. (2012) "Retos de las Facultades de Comunicación: Formación de nuevos perfiles de periodistas con base en la innovación y Tecnologías del conocimiento", en Revista de Tecnología Conocimiento y Sociedad. Illinois, USA Common Ground Publisher.

- $\quad$ Finberg, H. (2013) "Rethinking journalism education: A Call for Innovation”. Florida, EE.UU.

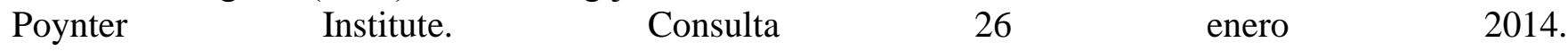
[http://www.newsu.org/course_files/StateOfJournalismEducation2013.pdf].

- $\quad$ Grimm, J. (2010) “Columbia's New Dual Degree Program Aims to Redefine Journalism As We Know it". Florida, USA. Poynter Institute. Consulta 3 febrero 2014 [http://www.poynter.org/howtos/career-development/ask-the-recruiter/104901/columbias-new-dual-degree-program-aims-toredefine-journalism-as-we-know-it/].

- $\quad$ Levinson, P. (2012) New New Media. 2nd. Edition. NY, USA. Penguin Academics

- $\quad$ Miravalls, J. (2014)’Periodismo, Escuela de oficio” en Ciberperiodismo, Lima, Perú. Ed. USMP Ed. USMP.

Ortega, F. (2014) “Adaptar la receta al cambio de paradigma” en Ciberperiodismo. Lima, Perú.

- $\quad$ Porto, D. y Flores, J. (2012) Periodismo Transmedia. Madrid, Fragua. enero 2014. 
[http://www.newsu.org/course_files/StateOfJournalismEducation2013.pdf]

Ribeiro Sombra, T, et al. (2020) Pattern Recognition in Scientific Social Networks: A Systematic Literature Review. In South Florida Journal of Development, Miami, v.1,n.2,22-36, apr./jun. 2020.ISSN 2675-5459

DOI:10.46932/sfjdv1n2-001

- Unesco (2007) Model curricula for journalism education. Colección de la UNESCO sobre los estudios de Periodismo. Consulta 3 de febrero de 2014.

[http://www.unesco.org/new/es/communication-and-information/resources/publications-andcommunication-materials/publications/full-list/model-curricula-for-journalism-education/] 